\title{
Co-option during the evolution of multicellular and developmental complexity in the volvocine green algae
}

3

Bradley J.S.C. Olson ${ }^{1 *}$ and Aurora M. Nedelcu²

Division of Biology

Kansas State University

Manhattan, KS USA

bjsco@ksu.edu

Department of Biology

University of New Brunswick

Fredericton, NB Canada

anedelcu@unb.ca

*Corresponding author (bjsco@ksu.edu)

Running title: Multicellularity by co-option in the Volvocales

Keywords: Multicellularity, Developmental complexity, Gene co-option, Volvocales, Volvox, Chlamydomonas, Gonium 


\section{Abstract}

Despite its major impact on the evolution of Life on Earth, the transition to multicellularity remains poorly understood, especially in terms of its genetic basis. The volvocine algae are a group of closely related species that range in morphology from unicellular individuals (Chlamydomonas) to undifferentiated multicellular forms (Gonium) and complex organisms with distinct developmental programs and one (Pleodorina) or two (Volvox) specialized cell types. Modern genetic approaches, complemented by the recent sequencing of genomes from several key species, revealed that co-option of existing genes and pathways is the primary driving force for the evolution of multicellularity in this lineage. The initial transition to undifferentiated multicellularity, as typified by the extant Gonium, was driven primarily by the co-option of cell cycle regulation. Further morphological and developmental innovations in the lineage leading to Volvox resulted from additional co-option events involving genes important for embryonic inversion, asymmetric cell division, somatic and germ cell differentiation and the structure and function of the extracellular matrix. Due to their relatively low but variable levels of morphological and developmental complexity, simple underlying genetics and recent evolutionary history, the volvocine algae are providing significant insight into our understanding of the genetics and evolution of major developmental and morphological traits.

\section{Introduction}

Multicellularity evolved independently at least twenty-five times, in both prokaryotic and eukaryotic clades [1-4], suggesting it is a common adaptation in response to various ecological pressures such as predation, nutrient limitation or changing environments [reviewed in 3-5]. The transition to simple multicellular life opened up unprecedented opportunities for the evolution of complex bodies with specialized cells and novel developmental plans. Most multicellular organisms, including plants and animals, develop from a single progenitor cell, a process known as clonal/unitary development (see $[3,6]$ for alternative developmental modes). Despite being one of the few major evolutionary transitions that shaped Life on Earth $[3,7,8]$, the genetic basis for the evolution of multicellularity has been elusive, partly because extant lineages and their genomes have changed significantly since diverging from their unicellular ancestors [9-12].

Generally, the evolution of new traits relies on two major processes; de novo gene evolution and co-option of existing genes for new functions [13-15]. The former is less understood, however, with more genomes being sequenced and more advanced computational approaches becoming available the role of de novo genes to the evolutionary process is being re-assessed $[13,14,16]$. Gene co-option can involve single-copy or duplicated genes. Both structural (i.e., coding for RNAs or proteins other than regulatory factors) and regulatory genes can be co-opted via changes in either their coding or regulatory sequences, or both $[13,14,17]$. Co-option can also involve a change in the binding spectrum of an existing protein by the virtue of a fortuitous interaction with a newly evolved protein, a process referred to as 'molecular exploitation' [18]. Co-option of existing genes has been often invoked to underlie the evolution of numerous adaptive traits, including those associated with the multicellular phenotype $[13,14,17,19]$. However, the relative significance of the postulated co-option mechanisms remains a matter of debate, with changes in gene regulation often considered the primary contributors to morphological evolution [19-22]. As genomics is transforming our understanding of the genetic basis of many processes, the mechanisms and sequence of events involved in the evolution of multicellular and developmental complexity are starting to become clear in several major multicellular lineages. Here we focus on recent advances in our understanding of multicellular 
evolution using the volvocine green algae as a model system, and argue that co-option of both regulatory and structural genes involving changes in regulatory as well as coding sequences played a major role in the evolution of morphological and developmental complexity in this group.

The volvocine algae, in the order Volvocales, include a series of species with morphologies ranging from unicellular forms such as in Chlamydomonas, to multicellular groups of unspecialized cells (e.g. Gonium and Eudorina), and to complex multicellular individuals with one (e.g. Pleodorina; somatic cells), or two (Volvox; somatic and germ cells) specialized cell types (Fig. 1). The Volvocales are an experimentally tractable model-system for understanding the mechanistic basis for the evolution of multicellular complexity for several reasons [5]. Multicellularity in the Volvocales occurred more recently, 200 Mya [23], than in other multicellular lineages; for instance, in the animal and land plant lineages multicellularity evolved -0.65-1 Bya $[9,10,24-26]$. The genomes of 3 volvocine species - Chlamydomonas reinhardtii, Gonium pectorale, and Volvox carteri - have been sequenced [27-29], and although these species span the range of complexity from unicellular to multicellular forms with simple or complex developmental programs, their genomes appear overall similar, with some of the differences likely to have contributed to the evolution of multicellular complexity in this group. Indeed, both genomics and genetics have revealed that the genetic basis for major leaps in developmental and morphological complexity in the Volvocales is rather simple [30-40]. Genetic tools are also available to tease apart the contributions of the various postulated genetic mechanisms underlying the evolution of morphological innovations [41-45]. Furthermore, genetics screens have been a powerful tool in advancing our understanding of developmental pathways in $\mathrm{V}$. carteri, where a number of mutants have revealed genes involved in important developmental and morphological traits [30-33,40,46,47]. Lastly, while the transition to multicellular undifferentiated forms occurred only once, several traits associated with organismal size expansion and developmental programs have been repeatedly gained and lost in this group $[12,23,41,48-51]$, opening up the possibility to address the genetic basis of morphological convergence.

\section{Co-option of cell cycle regulation during the evolution of undifferentiated multicellularity in volvocine algae}

In metazoans and plants, the regulation of the cell cycle involves a group of retinoblastomarelated proteins; these are transcriptional regulators that repress and activate cell cycle regulated genes through binding to, and directly affecting the activity of E2F-DP transcription factors [62]. When hypo-phosphorylated, RB proteins repress the cell cycle, but when hyperphosphorylated, primarily by cyclin-CDK dimers, RB becomes inactivated, thereby derepressing and activating $S$ phase-related transcription driving the cell into mitosis [62].

A recurring theme of multicellular evolution in the Volvocales involves modifications to their cell cycle program $[5,12,41,50]$ (Fig. 2). Our understanding of the volvocalean cell cycle is primarily derived from work in $C$. reinhardtii. In this species, each single-celled individual follows a multiple fission cell cycle, where cells grow $2^{n}$ in size, followed by $n$ alternating rounds of divisions. The cycle becomes highly synchronized with the diurnal light-dark cycle, resulting in a prolonged G1 period during light and a series of S-phase and mitosis (S/M) in the dark, to produce a uniform population of unicellular daughter cells (Fig. 2) [52-57]. Interestingly, in C. reinhardtii, the number of alternating rounds of S/M phase is determined by the mother cell size, which regulates the activity of the Chlamydomonas retinoblastoma protein, RB (encoded by the 
MAT3 gene), and determines the number of rounds of cell division after cells have reached a minimum size to divide at least once $[52,53,55,56,58]$ (Fig. 2).

Multiple fission has been co-opted for multicellularity in the Volvocales. While in unicellular Chlamydomonas individual daughters break apart from division clusters, in undifferentiated multicellular species such as Gonium failure to separate at the end of the process results in multicellular daughter colonies (Fig. 2). In V. carteri (Fig. 1) and its close relatives, clusters of undifferentiated cells ultimately undergo a series of asymmetric cell divisions resulting in the establishment of the germ and somatic cell lines [5,33,41,51,59-61]. Because RB is a key regulator of multiple fission and of the number of division cycles [52-54,56], it is likely that this gene has been important during the evolution of multicellularity in the volvocine lineage (Fig. 2).

In contrast to most plants and metazoans, which have multiple isoforms of RB, E2F and DP with unique and overlapping roles [62-65], C. reinhardtii, G. pectorale and V. carteri have single copies of RB, E2F and DP. However, the RB cell cycle regulatory pathway differs between the unicellular and multicellular volvocine algae $[27,66]$. The RB gene itself is regulated by dimers of two proteins, a cyclin and a cyclin-dependent kinase (CDK), which act as dimers to phosphorylate RB. Cyclin D1 plays a critical role in regulating the transition from $\mathrm{G} 1$ to $\mathrm{S}$ phase (Fig. 2). Interestingly, C. reinhardtii has a single cyclin D1 gene, while G. pectorale and V. carteri each have an expanded repertoire of cyclin D1 genes [27, 28]. Similarly, the linker domain of the RB protein - where cyclin-CDK dimers phosphorylate RB $[52,66]$, is different between the unicellular Chlamydomonas and the multicellular Gonium and Volvox [27]. Interestingly, RB genes in the Volvocales are also tightly linked to their mating type loci, likely because they have secondary roles in regulating the sex cycle [41,66-68]. Because the RB pathway regulates the transcription of cell cycle related genes, it is likely that the transcriptional output of the RB pathway has been co-opted for a role in multicellularity, and subsequently for cell differentiation, and sexual development [27].

Recently, this hypothesis has been directly tested by expressing the RB gene from $G$. pectorale in C. reinhardtii cells lacking the $R B$ gene [27]. Surprisingly, Chlamydomonas cells complemented with the Gonium RB gene exhibited a multicellular phenotype. When these lines were crossed to mutants lacking a functional E2F-DP transcription factor, this phenotype was suppressed [27]. These data support the hypothesis that RB-dependent regulation of existing cell adhesion genes present in the unicellular ancestor was important for multicellular evolution in this group. Because RB proteins are central to cell cycle regulation and development in many eukaryotes [69-75], this finding has significant implications for our understanding of the evolution of multicellularity in other eukaryotic taxa as well.

\section{Co-option of environmentally-induced stress responses for somatic cell differentiation in V. carteri}

Generally, the evolution of germ-soma separation during the transition to multicellularity requires a change in the expression of vegetative and reproductive functions from a temporal pattern into a spatial context - resulting in these functions being differentially expressed between somatic and germ cells [76]. Mechanistically, it has been suggested that the evolution of soma involved the co-option of life-history genes that in unicellular lineages were induced by environmental cues as an adaptive strategy to enhance survival at an immediate cost to reproduction, by shifting their expression from an environmentally-induced context into a developmental context $[76,77]$. 
In $V$. carteri, the segregation between the somatic and germ cell lines takes place early during embryonic development and involves a series of asymmetric cell divisions limited to one hemisphere of the embryo. Differences in cell size, not cytoplasmic composition, are thought to be solely responsible for the early establishment of distinct cell fates in this species [59]. Somatic cell specialization involves the differential expression of a master regulatory gene known as regA, thought to encode a transcriptional repressor; mutations in this gene alone result in somatic cells regaining reproductive capabilities $[47,78]$. The proposed DNA binding domain of RegA is a SAND domain that is also found in other transcription factors such as ULTRAPETALA in Arabidopsis thaliana and SP100 in humans, where they are involved in regulating cell proliferation and differentiation $[79,80]$. RegA is only induced in cells whose size falls below a threshold size at the end of embryogenesis; the mechanism is unknown, but likely involves cis-regulatory elements identified in three of its intronic sequences, [34,59]. RegA is thought to act by repressing the expression of nuclear-encoded chloroplast proteins [81], which affects the ability of these small cells to photosynthesize, and ultimately to grow and divide.

RegA belongs to a large and diverse gene family known as the VARL gene family with members in both unicellular and multicellular volvocine species [77,82-84]. Interestingly, orthologs of regA have been identified in several distant Volvox species that evolved somatic cell differentiation independently suggesting that regA originated before the evolution of somatic cell differentiation, although whether regA is involved in somatic cell differentiation in these Volvox species is not known [82,84]. However, a direct ortholog of regA has not been found in either $C$. reinhardtii or $G$. pectorale genomes [27,84]. The closest homolog of regA in $C$. reinhardtii is known as RLS1 and its expression is induced under nutrient limitation (including phosphorusand sulfur-deprivation), light deprivation and during stationary phase $[77,85]$. Based on these findings we proposed a hypothesis for the evolution of somatic cells in $V$. carteri involving the co-option of an ancestral environmentally-induced $R L S 1$-like gene, by switching its regulation from a temporal/environmental into a spatial/developmental context $[77,85]$.

Three potential scenarios, each with distinct predictions, can be envisioned for such a change in regulation (Fig. 3) [86]. In the first scenario, no new regulatory elements evolved; rather, the same ancestral environmentally-induced signaling pathway was also induced during development in early multicellular volvocine algae with somatic cells (that is, the developmental signal simulated the environmental signal) (Fig. 3A) [85]. This scenario would predict that regA in $V$. carteri is induced both environmentally and developmentally via the same signaling pathway (Fig. 3A). A second scenario requires that an additional layer of regulation evolved as part of a new, developmentally-induced signaling pathway, and both mechanisms have been maintained in $V$. carteri. This would predict that regA is induced both environmentally and developmentally, but the signaling pathways are different (Fig. 3B). The third scenario assumes that the ancestral regulation of $\operatorname{reg} A$ was replaced or lost and new regulatory elements evolved in $V$. carteri (Fig. 3C). Notably, the former two scenarios predict that regA can still be induced in an environmental context. Indeed, regA is now known to be expressed outside its developmental context, in response to environmental stresses such as light following extended darkness [87]. This suggests that regA maintained some of is putative ancestral environmental regulation. However, the current data cannot distinguish between the first two scenarios presented above (Fig. 3).

Understanding how an environmentally-induced $R L S 1$-like gene was co-opted into a regulator of cell differentiation will provide new insights into the evolution of novel morphological traits. In addition, understanding how a pathway involved in responses to environmental changes has 
been co-opted into a developmental program will contribute to the growing interest in reevaluating the role of environment in developmental evolution [88].

\section{Co-option of structural genes for developmental complexity in the Volvocales}

The two major leaps in organismal complexity discussed thus far, multicellularity and somatic cell differentiation, involved the co-option of transcriptional regulators, all of which are likely to impact the expression of many downstream genes. However, structural genes whose products are known to be involved in specific developmental and morphological traits in $V$. carteri have also been co-opted from single-celled ancestors. For example, four of the genes underlying two $V$. carteri specific developmental processes, asymmetric division and embryonic inversion, have orthologs in C. reinhardtii and G. pectorale (Table 1). Remarkably, V. carteri mutants in two of these genes, invA coding for a kinesin [40], and glsA coding for a co-chaperone involved in spindle placement $[33,60,89]$ can be complemented by their $C$. reinhardtii orthologs $[40,90]$, suggesting that these genes have been directly co-opted into developmental processes by either changes in their regulation (involving cis or trans elements) or changes in their protein interacting partners. Other structural genes involved in multicellular traits in the volvocine algae appear to have evolved by gene duplication followed by diversification; these include the genes coding for matrix metalloproteases (MMPs) and a class of hydroxyproline-rich glycoproteins called pherophorins (PHERs), all involved in the structure and function of the extracellular matrix (ECM) [27,28,91-96] (Table 1). Interestingly, although C. reinhardtii and G. pectorale have roughly the same total number of these ECM related genes (Table 1; though not all are direct orthologs to each other), $V$. carteri has significantly more ECM related genes than Chlamydomonas (Table 1; $[41,91]$ ), consistent with an increased amount of ECM in this species [27].

\section{Conclusions and Perspective}

The volvocine algae are reaping significant advances in our understanding of the genetic mechanisms underlying the evolution of multicellularity and developmental complexity. Several key traits associated with the evolution of multicellularity and developmental complexity in this group involved co-option events (Table 1). First, the cell cycle was reprogrammed via co-option of $R B$ and cyclin $\mathrm{D} 1$ genes to promote the evolution of undifferentiated multicellularity by modifications to the multiple fission division pattern as observed in the extant Gonium (Fig. 2, Table 1). Interestingly, the ancestral multiple fission type of division has been further modified in distinct Volvox lineages, contributing to the four developmental programs known in this group [97]. In the lineage leading to $V$. carteri, a series of additional co-option events took place, including co-option of genes involved in the structure and function of ECM, embryonic inversion, asymmetric cell division, and establishing the somatic cell fate (Fig. 1, Table 1). Of particular interest is the co-option of $\operatorname{reg} A$ in the differentiation of soma. Because soma evolved independently in several volvocine lineages $[23,41,48,97]$, including species whose developmental programs do not involve asymmetric divisions and multiple fission, further sequencing of volvocalean genomes and genetic analyses should reveal whether somatic cell evolution involved similar or distinct genetic mechanisms in this group.

The Volvocales are emerging as an important model-system in which to address the contribution of the many postulated types of genetic mechanisms contributing to the evolution of multicellularity and developmental complexity (Fig. 1, Table 1). Indeed, some co-option events involved changes in the regulation of genes (e.g., regA, CYCD1) while others involved changes 
1 to coding sequences (e.g., $R B$ ) or possibly the binding potential of the encoded proteins (e.g., 2 glsA, invA). Likewise, the co-option events included both single-copy genes (inv, glsA) as well

3 as multi-copy genes (VARLs, PHERs, MMPs); and both regulatory (regA, RB, CYCD1) and

4 structural (invA, glsA, PHER, MMP) genes. Due to their relatively low but variable levels of

5 complexity as well as simple underlying genetics and recent evolutionary history, the volvocine

6 algae are living up to their potential by providing significant insight into our understanding of the

7 genetics of adaptations and evolution of complex developmental and morphological traits.

Acknowledgements

10 This work was supported by the US National Science Foundation (MCB-1412738) to B.J.S.C.O and the Natural Sciences and Engineering Research Council of Canada to A.M.N. 


\begin{abstract}
1 Tables
3 Table 1: Summary of our current understanding of the genes involved in major morphological and developmental traits in volvocine 4 algae and the potential genetic mechanisms underlying their evolution. For gene abbreviations see text.
\end{abstract}

6

Species

Chlamydomonas reinhardtii

Gonium pectorale

Volvox carteri

Co-opted

gene

Likely change

underlying the co-option

event

\begin{tabular}{|c|c|c|c|c|c|c|c|c|c|c|c|c|}
\hline \multicolumn{3}{|c|}{ Multicellularity } & \multicolumn{3}{|c|}{ ECM } & \multicolumn{2}{|c|}{ Complete Inversion } & \multicolumn{2}{|c|}{ Asymmetric Division } & \multicolumn{3}{|c|}{ Somatic Cells } \\
\hline \multirow[t]{2}{*}{ Presence } & \multicolumn{2}{|c|}{ Genes } & \multirow[t]{2}{*}{ Presence } & \multicolumn{2}{|c|}{ Genes } & \multirow[t]{2}{*}{ Presence } & \multirow{2}{*}{$\begin{array}{c}\text { Genes } \\
\text { invA, } B, C\end{array}$} & \multirow[t]{2}{*}{ Presence } & \multirow{2}{*}{$\begin{array}{l}\text { Gene } \\
\text { glsA }\end{array}$} & \multirow[t]{2}{*}{ Presence } & \multicolumn{2}{|c|}{ Genes } \\
\hline & $R B$ & CYCD1 & & PHERs & MMPs & & & & & & VARLS & $\operatorname{reg} A$ \\
\hline $\mathrm{N}$ & 1 & 1 & $\mathrm{~N}$ & 31 & 44 & $\mathrm{~N}$ & 1 & $\mathrm{~N}$ & 1 & $\mathrm{~N}$ & 12 & 0 \\
\hline Y & 1 & 4 & $\mathrm{~N}$ & 35 & 36 & $\mathrm{~N}$ & 1 & $\mathrm{~N}$ & 1 & $\mathrm{~N}$ & 8 & 0 \\
\hline & Single copy & Duplicated & & Duplicated & & & $\begin{array}{l}\text { Single } \\
\text { copies }\end{array}$ & & Single copy & & Duplicate & \\
\hline & Coding & $\begin{array}{l}\text { Regulatory } \\
\text { or binding } \\
\text { partner }\end{array}$ & & Coding anc & ulatory & & $\begin{array}{l}\text { Regulatory } \\
\text { or in } \\
\text { binding } \\
\text { partners }\end{array}$ & & $\begin{array}{l}\text { Regulatory } \\
\text { or in binding } \\
\text { partners }\end{array}$ & & Regulatory & \\
\hline
\end{tabular}

7 


\section{Figure Legends}

Fig 1: Evolution of multicellular and developmental complexity in the Volvocales. Representative volvocine species with distinct levels of complexity, from single-celled individuals (Chlamydomonas) to complex multicellular organisms with two specialized cell types (Volvox). Genes that have been co-opted for novel morphological and developmental traits in this group have been mapped according to the current understanding of their evolutionary history (see Table 1 for more information).

Fig 2: Comparison between the cell cycles of the unicellular Chlamydomonas and the multicellular Gonium (A) Growth and cell division in Chlamydomonas are coupled such that after an extended G1 phase in the light, they divide by multiple-fission in the dark. Chromatin bound RB-E2F-DP complexes transcriptionally regulate the multiple-fission cell cycle. During G1 phase, RB is hypo-phosphorylated and binds to, and inhibits transcription by, E2F-DP. During mitosis, cyclin D1 dimerized with a CDK hyper-phosphorylates RB, causing a conformational change in the chromatin bound complex, leading to the transcription of genes required for mitosis. After mitosis, RB becomes hypo-phosphorylated and cell cycle related genes are no longer transcribed, causing mitotic exit. (B) Gonium divides by multiple fission as well. RB also regulates the Gonium cell cycle except that during G1 phase the several cyclin D1 genes and their CDK partner phosphorylate RB at loci different than in Chlamydomonas, regulating genes required for cell-cell adhesion during G1 phase. During mitosis, cyclin D1 family genes with their CDK partner activate the cell cycle just as in Chlamydomonas; however, after mitosis, genes required for $\mathrm{G} 1$ phase cell-cell adhesion are up-regulated just before mitosis ends, keeping post-mitotic cells attached to each other and leading to the production of multicellular daughter colonies.

Fig 3: Three potential scenarios for the co-option of an ancestral, environmentallyinduced $\operatorname{reg} A$-like gene during the evolution of reg $A$ in the lineage leading to $V$. carteri. (A) No new regulatory elements (RE) evolved; both environmental and developmental cues converge on the same signal and ancestral regulatory element (RE1). (B) An additional layer of regulation (RE2) evolved as part of a new (developmentally-induced) signaling pathway. (C) The ancestral regulation was replaced or lost and new regulatory elements (RE2) evolved. 


\section{References}

3 1. Baldauf SL: The deep roots of eukaryotes. Sci. (New York, NY) 2003, 300:1703.

4 2. King N: The unicellular ancestry of animal development. Dev. Cell 2004, 7:313-325.

$53 . \quad{ }^{* *}$ Grosberg RK, Strathmann RR: The evolution of multicellularity: a minor major

6 transition? Annu. Rev. Ecol. Evol. Syst. 2007, 38:621.

7 A comprehensive overview of multicellularity, including several developmental modes.

9 4. Bonner JT: First Signals. Princeton University Press; 2000.

5. Kirk DL: Volvox: molecular-genetic origins of multicellularity and cellular differentiation. Cambridge University Press; 1998.

12 6. Olson BJ: From brief encounters to lifelong unions. Elife 2013, 2:e01893.

13 7. ${ }^{\star *}$ Szathmáry E: Toward major evolutionary transitions theory 2.0. Proc. Natl. Acad.

14 Sci. 2015, doi:10.1073/pnas.1421398112.

15 An update of the major transitions in evolution.

8. Maynard Smith J, Szathmáry E: The Major Transitions in Evolution. Oxford University Press; 1995.

9. *King N, Westbrook MJ, Young SL, Kuo A, Abedin M, Chapman J, Fairclough S, Hellsten $U$, Isogai $Y$, Letunic I, et al.: The genome of the choanoflagellate Monosiga brevicollis and the origin of metazoans. Nature 2008, 451:783-788.

22 The genome of the choanoflagellate of Monosiga brevicolis, a unicellular outgroup to Metazoa.

10. *Suga H, Chen Z, de Mendoza A, Sebé-Pedrós A, Brown MW, Kramer E, Carr M, Kerner $P$, Vervoort M, Sánchez-Pons N, et al.: The Capsaspora genome reveals a complex unicellular prehistory of animals. Nat. Commun. 2013, 4:2325.

27 This paper reports the genome of Capsaspora, a unicellular outgroup to Metazoa, and 28 discusses evidence of gene co-option during the evolution of multicellularity.

11. Parfrey LW, Lahr DJG: Multicellularity arose several times in the evolution of eukaryotes (Response to DOI 10.1002/bies. 201100187). Bioessays 2013, 34:833-840. doi:10.1002/bies.201100187.

33 12. Kirk DL: A twelve-step program for evolving multicellularity and a division of labor. 
BioEssays 2005, 27:299-310.

\section{Zhou Q, Wang W: On the origin and evolution of new genes-a genomic and} experimental perspective. J. Genet. Genomics 2008, 35:639-648.

14. *Kaessmann H: Origins, evolution, and phenotypic impact of new genes. Genome Res. 2010, 20:1313-1326.

A comprehensive overview of the origin of new genes, including mechanisms of co-option.

15. Taylor JS, Raes J: Duplication and divergence: the evolution of new genes and old ideas. Annu. Rev. Genet. 2004, 38:615-43.

16. Tautz D, Domazet-Loso T: The evolutionary origin of orphan genes. Nat. Rev. Genet. 2011, 12:692-702.

17. $\quad$ True JR, Carroll SB: Gene co-option in physiological and morphological evolution. Annu. Rev. Cell Dev. Biol. 2002, 18:53-80.

Provides a framework for understanding the origin of co-optive evolution and the mechanisms that promote evolutionary novelty by re-using the genetic toolkit.

18. Bridgham JT: Evolution of Hormone-Receptor Complexity by Molecular Exploitation. Science. 2006, 312:97-101.

19. ${ }^{*}$ Carroll SB: Evo-Devo and an Expanding Evolutionary Synthesis: A Genetic Theory of Morphological Evolution. Cell 2008, 134:25-36.

Outlines eight principles derived from molecular and evolutionary developmental biology that have led to a genetic theory of morphological evolution.

20. ${ }^{*}$ Carroll SB: Endless forms: the evolution of gene regulation and morphological diversity. Cell 2000, 101:577-580.

Reviews the body of evidence that points to a central role for differences in developmental gene regulation in both intraspecific variation and the diversification of body plans and body parts

21. *Hoekstra HE, Coyne JA: The locus of evolution: Evo devo and the genetics of adaptation. Evolution (N. Y). 2007, 61:995-1016.

Discusses and critiques the assertion that adaptive mutations affecting morphology are more 
2 22. Wittkopp PJ, Kalay G: Cis-regulatory elements: molecular mechanisms and

3 evolutionary processes underlying divergence. Nat. Rev. Genet. 2011, 13:59-69.

4 23. ${ }^{* *}$ Herron MD, Hackett JD, Aylward FO, Michod RE: Triassic origin and early radiation

5 of multicellular volvocine algae. Proc. Natl. Acad. Sci. 2009, 106:3254-3258.

6 Mapping of David Kirk's twelve steps on the Volvocales phylogeny.

24. Suga H, Ruiz-Trillo I: Development of ichthyosporeans sheds light on the origin of metazoan multicellularity. Dev. Biol. 2013, 377:284-92.

25. Parfrey LW, Lahr DJG, Knoll AH, Katz LA: Estimating the timing of early eukaryotic diversification with multigene molecular clocks. Proc. Natl. Acad. Sci. U. S. A. 2011, 108:13624-9.

26. Hori K, Maruyama F, Fujisawa T, Togashi T, Yamamoto N, Seo M, Sato S, Yamada T, Mori $\mathrm{H}$, Tajima $\mathrm{N}$, et al.: Klebsormidium flaccidum genome reveals primary factors for plant terrestrial adaptation. Nat. Commun. 2014, 5:1-9.

27. ${ }^{\star *}$ Hanschen ER, Marriage TN, Ferris PJ, Hamaji T, Toyoda A, Fujiyama A, Neme R, Noguchi $H$, Minakuchi $Y$, Suzuki M, et al.: The Gonium pectorale genome demonstrates cooption of cell cycle regulation for multicellularity. Nat. Commun. 2016, 7:11370.

The Gonium pectorale genome sequence and experimental evidence that RB plays a key role in

28. **Prochnik SE, Umen J, Nedelcu AM, Hallmann A, Miller SM, Nishii I, Ferris P, Kuo A, Mitros T, Fritz-Laylin LK, et al.: Genomic analysis of organismal complexity in the multicellular green alga Volvox carteri. Science 2010, 329:223-226.

A comparative analysis of Volvox carteri and Chlamydomonas reinhardtii genomes revealed a small number of differences in their genomes, in spite of major differences in morphological complexity between the two volvocine algae.

29. Merchant SS, Prochnik SE, Vallon O, Harris EH, Karpowicz SJ, Witman GB, Terry A, Salamov A, Fritz-Laylin LK, Maréchal-Drouard L, et al.: The Chlamydomonas genome reveals the evolution of key animal and plant functions. Science 2007, 318:245-250.

30. Huskey RJ, Griffin BE: Genetic control of somatic cell differentiation in Volvox analysis of somatic regenerator mutants. Dev. Biol. 1979, 72:226-235. 
70:1335-1338.

2 32. Starr RC, Jaenicke L: Cell differentiation in Volvox carteri (Chlorophyceae): the use of mutants in understanding patterns and control. Plant Biol. 1989,

33. Miller SM, Kirk DL: glsA, a Volvox gene required for asymmetric division and germ cell specification, encodes a chaperone-like protein. Development 1999, 126:649658.

34. Stark K, Kirk DL, Schmitt R: Two enhancers and one silencer located in the introns of regA control somatic cell differentiation in Volvox carteri. Genes \&amp; Dev. 2001, 15:1449-1460.

35. Huskey RJ: Mutants affecting vegetative cell orientation in Volvox carteri. Dev. Biol. 1979, 72:236-43.

36. Kurn N, Colb M, Shapiro L: Spontaneous frequency of a developmental mutant in Volvox. Dev. Biol. 1978, 66:266-269.

37. Kirk DL, Kaufman MR, Keeling RM, Stamer KA: Genetic and cytological control of the asymmetric divisions that pattern the Volvox embryo. Development Suppl. 1990, 1:67-82.

38. Huskey R, Griffin B, Cecil P, Callahan A: A Preliminary Genetic Investigation of Volvox Carteri. Genetics 1979, 91:229-244.

39. Tam LW, Kirk DL: The program for cellular differentiation in Volvox carteri as revealed by molecular analysis of development in a gonidialess/somatic regenerator mutant. Development 1991, 112:571-580.

Nishii I, Ogihara S, Kirk DL: A kinesin, invA, plays an essential role in volvox morphogenesis. Cell 2003, 113:743-753.

41. Umen JG, Olson BJSC: Genomics of Volvocine Algae. In Advances in Botanical

42. Lerche $\mathrm{K}$, Hallmann A: Stable nuclear transformation of Eudorina elegans. $B M C$

43. Lerche K, Hallmann A: Stable nuclear transformation of Gonium pectorale. BMC Biotechnol. 2009, 9:64.

44. Hallmann A, Rappel A: Genetic engineering of the multicellular green algaVolvox: a modified and multiplied bacterial antibiotic resistance gene as a dominant selectable marker. Plant J. 1999, 17:99-109. transformation of Volvox carteri. Proc. Natl. Acad. Sci. U. S. A. 1994, 91:5080-5084. 
47. Kirk DL, Baran GJ, Harper JF, Huskey RJ, Huson KS, Zagris N: Stage-specific hypermutability of the regA locus of Volvox, a gene regulating the germ-soma dichotomy. Cell 1987, 48:11-24.

50.

Nozaki H, Misawa K, Kajita T, Kato M, Nohara S, Watanabe MM: Origin and evolution of the colonial volvocales (Chlorophyceae) as inferred from multiple, chloroplast gene sequences. Mol. Phylogenet. Evol. 2000, 17:256-268.

49. Nozaki H, Yamada TK, Takahashi F, Matsuzaki R, Nakada T: New "missing link" genus of the colonial volvocine green algae gives insights into the evolution of oogamy. BMC Evol. Biol. 2014, 14:37.

*Coleman AW: A Comparative Analysis Of The Volvocaceae (Chlorophyta). J. Phycol. 2012, 48:491-513.

Provides an up-to-date review of Volvocales.

51. Nishii I, Miller SM: Volvox: simple steps to developmental complexity? Curr. Opin. Plant Biol. 2010, 13:646-653.

52. Olson B, Oberholzer M, Li Y, Zones JM, Kohli HS, Bisova K, Fang S-C, Meisenhelder J, Hunter T, Umen JG: Regulation of the Chlamydomonas cell cycle by a stable, chromatin-associated retinoblastoma tumor suppressor complex. Plant Cell 2010, 22:3331-3347.

53. Umen JG, Goodenough UW: Control of cell division by a retinoblastoma protein homolog in Chlamydomonas. Genes Dev. 2001, 15:1652.

54. Bisova K, Krylov DM, Umen JG: Genome-wide annotation and expression profiling of cell cycle regulatory genes in Chlamydomonas reinhardtii. Plant Physiol 2005, 137:475-491.

55. Umen JG: The elusive sizer. Curr. Opin. Cell Biol. 2005, 17:435-41.

56. Fang S-C, de los Reyes C, Umen JG: Cell size checkpoint control by the retinoblastoma tumor suppressor pathway. PLoS Genet. 2006, 2:e167.

57. Harper J, John P: Coordination of division events in the Chlamydomonas cell cycle. Protoplasma 1986, 131:118-130.

58. Fang S-C, Umen JG: A suppressor screen in chlamydomonas identifies novel components of the retinoblastoma tumor suppressor pathway. Genetics 2008, 178:1295-1310.

59. Kirk MM, Ransick A, McRae SE, Kirk DL: The relationship between cell size and cell fate in Volvox carteri. J. Cell Biol. 1993, 123:191-208.

60. Pappas V, Miller SM: Functional analysis of the Volvox carteri asymmetric division 
protein GIsA. Mech. Dev. 2009, 126:842-851.

61. Herron MD, Desnitskiy AG, Michod RE: Evolution Of Developmental Programs In Volvox (Chlorophyta). J. Phycol. 2010, 46:316-324.

62. van den Heuvel S, Dyson $\mathrm{NJ}$ : Conserved functions of the pRB and E2F families. Nat. Rev. Mol. Cell Biol. 2008, 9:713-724.

63. Lammens T, Li J, Leone G, de Veylder L: Atypical E2Fs: new players in the E2F transcription factor family. Trends Cell Biol. 2009, 19:111-118.

64. Rowland BD, Bernards R: Re-evaluating cell-cycle regulation by E2Fs. Cell 2006, 127:871-874.

65. Inzé D: Green light for the cell cycle. EMBO J. 2005, 24:657-662.

66. *Ferris PJ, Olson B, de Hoff PL, Douglass S, Casero D, Prochnik SE, Geng S, Rai R, Grimwood J, Schmutz J, et al.: Evolution of an expanded sex-determining locus in Volvox. Sci. (New York, NY) 2010, 328:351-354.

The paper analysis the Volvox carteri male and female mating-type loci and discusses how the sex loci co-opted genes for male and female sexual programs in $V$. carteri compared to $C$. reinhardtii

67. *de Hoff PL, Ferris P, Olson B, Miyagi A, Geng S, Umen JG: Species and Population Level Molecular Profiling Reveals Cryptic Recombination and Emergent Asymmetry in the Dimorphic Mating Locus of C. reinhardtii. PLoS Genet. 2013, 9:e1003724.

Analysis of the C. reinhardtii plus and minus mating type loci demonstrating the evolutionary

69. Khidr L, Chen P-L: RB, the conductor that orchestrates life, death and differentiation. Oncogene 2006, 25:5210-5219.

71. Polager S, Ginsberg D: E2F - at the crossroads of life and death. Trends Cell Biol. 2008, 18:528-535. 
73. McClellan KA, Slack RS: Specific in vivo roles for E2Fs in differentiation and development. Cell cycle 2007, 6:2917-2927.

74. Knudsen ES, Knudsen KE: Retinoblastoma tumor suppressor: where cancer meets the cell cycle. Exp. Biol. Med. 2006, 231:1271-1281.

75. Manning AL, Dyson NJ: pRB, a tumor suppressor with a stabilizing presence. Trends Cell Biol. 2011, 21:433-441.

76. Nedelcu AM, Michod RE: Evolvability, Modularity, and Individuality during the Transition to Multicellularity in Volvacalean Green Algae. In Modularity in development and evolution. Edited by Schlosser G, Wagner G E. University Of Chicago Press; 2004:466-489.

77. Nedelcu AM, Michod RE: The evolutionary origin of an altruistic gene. Mol. Biol. Evol. 2006, 23:1460-1464.

78. Kirk MM, Stark K, Miller SM, Müller W, Taillon BE, Gruber H, Schmitt R, Kirk DL: regA, a Volvox gene that plays a central role in germ-soma differentiation, encodes a novel regulatory protein. Development 1999, 126:639-647.

79. Bottomley MJ, Collard MW, Huggenvik JI, Liu Z, Gibson TJ, Sattler M: The SAND domain structure defines a novel DNA-binding fold in transcriptional regulation. Nat. Struct. Biol. 2001, 8:626-633.

80. Carles CC, Fletcher JC: Missing links between histones and RNA Pol II arising from SAND? Epigenetics 2010, 5:381-385.

81. Meissner M, Stark K, Cresnar B, Kirk DL, Schmitt R: Volvox germline-specific genes that are putative targets of RegA repression encode chloroplast proteins. Curr. Genet. 1999, 36:363-370.

82. Duncan L, Nishii I, Howard A, Kirk D, Miller SM: Orthologs and paralogs of regA, a master cell-type regulatory gene in Volvox carteri. Curr. Genet. 2006, 50:61-72.

83. ${ }^{*}$ Duncan L, Nishii I, Harryman A, Buckley S, Howard A, Friedman NR, Miller SM: The VARL gene family and the evolutionary origins of the master cell-type regulatory gene, regA, in Volvox carteri. J. Mol. Evol. 2007, 65:1-11.

The paper reports additional regA-like genes in Chlamydomonas and two strains of Volvox, and provides an extensive analysis of the VARL domain.

84. ${ }^{* *}$ Hanschen ER, Ferris PJ, Michod RE: Early evolution of the genetic basis for soma in the volvocaceae. Evolution 2014, 68:2014-25.

34 This paper provides evidence that regA evolved in the Volvocales earlier than anticipated. 
altruism. Biol. Lett. 2009, 5:805-808.

86. König SG, Nedelcu AM: The mechanistic basis for the evolution of soma during the transition to multicellularity in the volvocine algae. In Multicellularity: Origins and Evolution. Edited by Niklas K, Newman S. MIT Press; 2016: 43-70

88. Moczek AP: Re-evaluating the environment in developmental evolution. Front. Ecol. Evol. 2015, 3:1-8.

89. Cheng Q, Fowler R, Tam L, Edwards L, Miller SM: The role of GlsA in the evolution of asymmetric cell division in the green alga Volvox carteri. Dev. Genes Evol. 2003, 213:328-335.

90. Cheng Q, Pappas V, Hallmann A, Miller SM: Hsp70A and GIsA interact as partner chaperones to regulate asymmetric division in Volvox. Dev. Biol. 2005, 286:537-548.

91. Kirk DL, Birchem R, King N: The extracellular matrix of Volvox: a comparative study and proposed system of nomenclature. J. Cell Sci. 1986, 80:207-231.

92. Hallmann A: The pherophorins: common, versatile building blocks in the evolution of extracellular matrix architecture in Volvocales. Plant J. 2006, 45:292-307.

93. Sumper M, Nink J, Wenzl S: Self-assembly and cross-linking of Volvox extracellular matrix glycoproteins are specifically inhibited by Ellman's reagent. Eur. J. Biochem. 2000, 267:2334-9.

94. Ertl H, Mengele R, Wenzl S, Engel J, Sumper M: The extracellular matrix of Volvox carteri: molecular structure of the cellular compartment. J. Cell Biol. 1989, 109:3493-3501.

95. Sumper M, Hallmann A: Biochemistry of the extracellular matrix of Volvox. Int. Rev. Cytol. 1997, 180:51-85.

96. Huber O, Sumper M: Algal-CAMs: isoforms of a cell adhesion molecule in embryos of the alga Volvox with homology to Drosophila fasciclin I. EMBO J. 1994, 13:42124222.

97. Desnitski AG: A Review On The Evolution Of Development In Volvox-Morphological And Physiological Aspects. Eur. J. Protistol. 1995, 31:241-247. 
Morphological or

Developmental Traits

Unicellular

Multiple fission

Undifferentiated multicellular Multiple fission

Undifferentiated multicellular Multiple fission

Expanded ECM

Embryo inversion

Differentiated multicellular

One specialized cell type: Soma

Multiple fission

Expanded ECM

Embryo inversion

Differentiated multicellular

Two specialized cell types:

Soma and germ

Multiple fission

Expanded ECM

Embryo inversion

Asymmetric cell division
Co-opted genes

$R B$

CYCD1

Unknown

invA

gls $A$

PHERs

MMPS

VARLs

regA

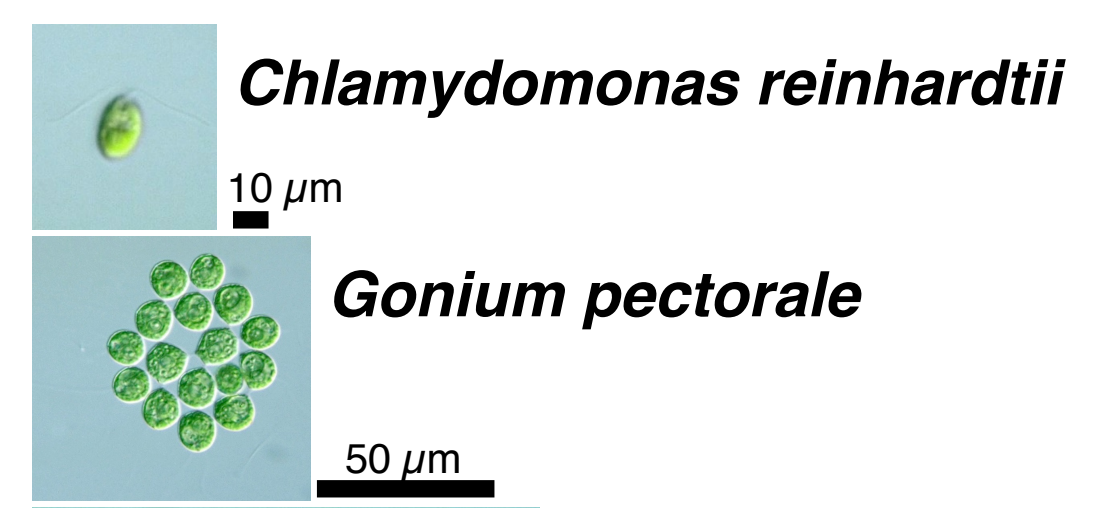

Eudorina elegans

$0.1 \mathrm{~mm}$

Pleodorina starii

$0.1 \mathrm{~mm}$

Volvox

carteri

$0.1 \mathrm{~mm}$ 


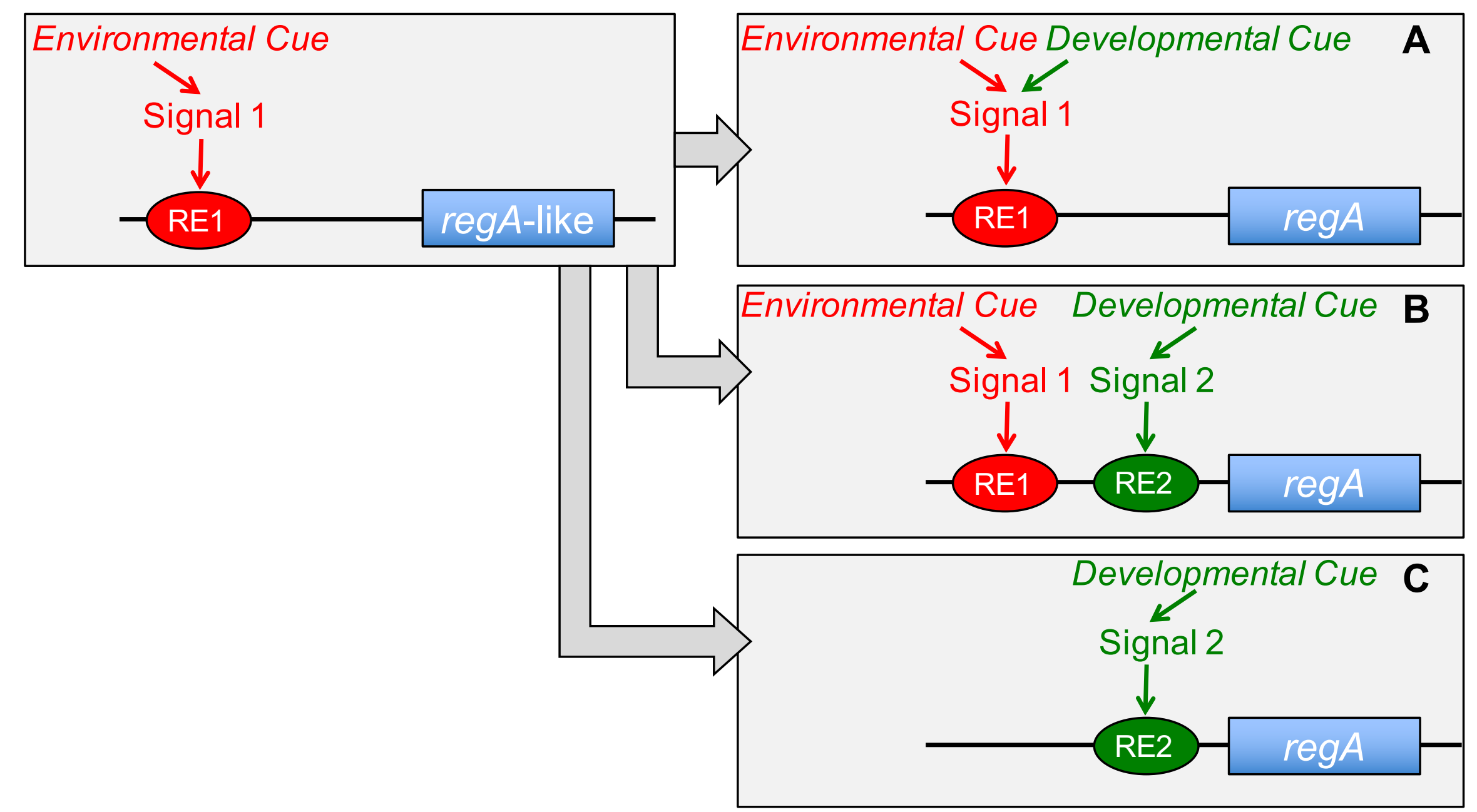




\section{\begin{tabular}{|ll}
\hline Early G1 Late G1 $>$ Multiple Fission & Post-Mitotic \\
\hline
\end{tabular}}

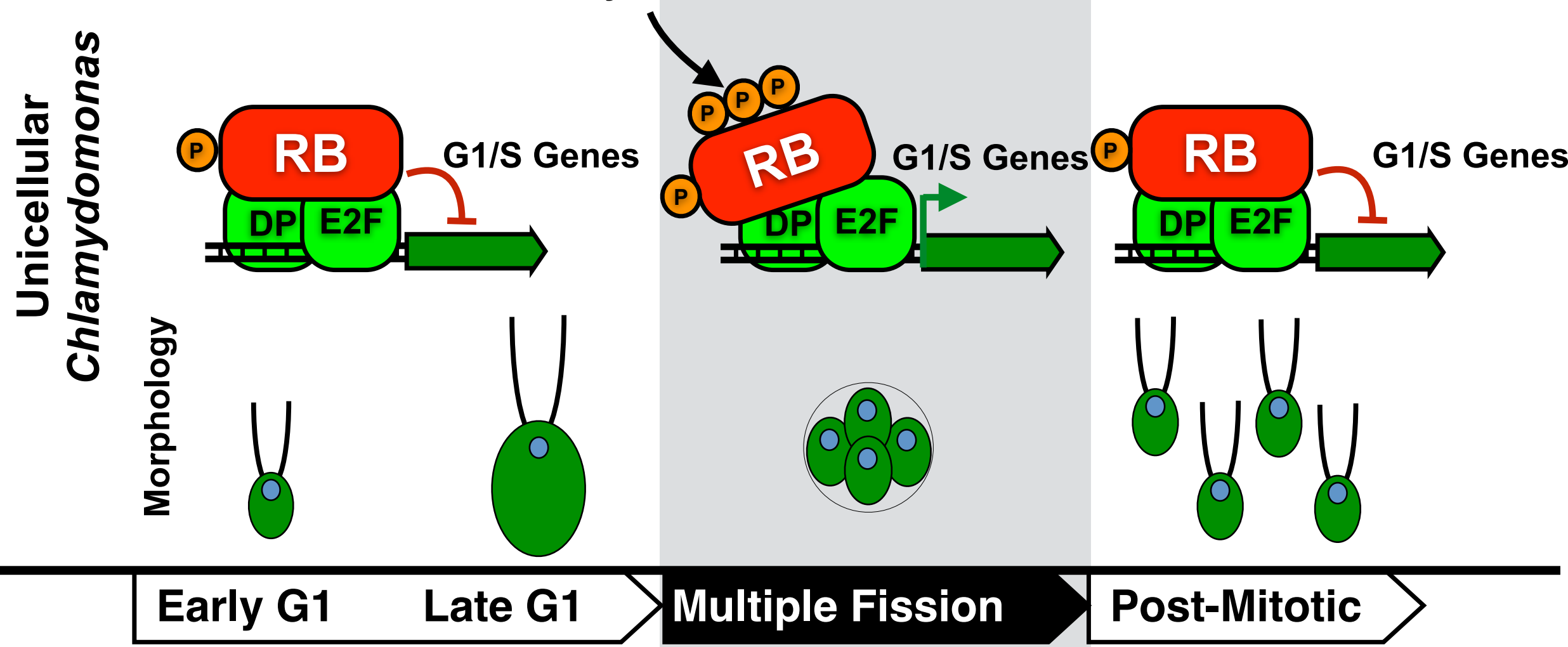

$$
\text { CycD1.x/CDK }
$$
Early G1 Late G1
Cy

CycD1.x/CDK
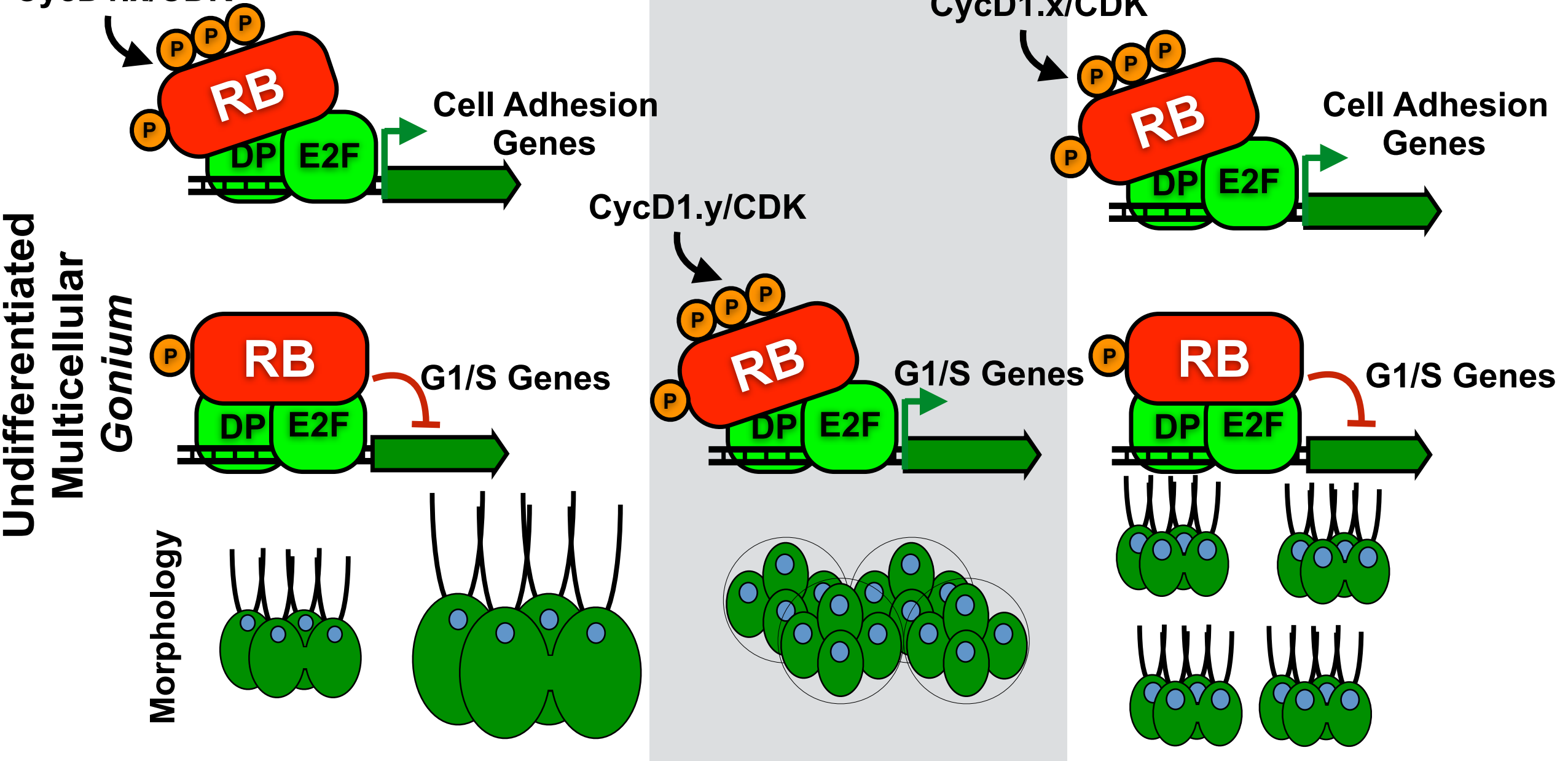